Asia Pacific Journal of Mathematics, Vol. 5, No. 2 (2018), 219-225

ISSN 2357-2205

\title{
STABILITY ANALYSIS OF PARTIAL DIFFERENTIAL EQUATIONS WITH PIECEWISE CONSTANT ARGUMENTS
}

\author{
QI WANG*, ZITING LIU \\ School of Applied Mathematics, Guangdong University of Technology, Guangzhou, 510006, P.R. China \\ Corresponding author: bmwzwq@126.com \\ Received May 28, 2018
}

\begin{abstract}
In this paper, the analytical stability of a partial differential equation with piecewise constant arguments is considered. By using the theory of separation of variables in matrix form and the Fourier method, the sufficient conditions under which the analytic solution is asymptotically stable are achieved. The results extend some existing ones. 2010 Mathematics Subject Classification. 35B35; 35F40.
\end{abstract}

Key words and phrases. partial differential equation; piecewise constant arguments; asymptotic stability.

\section{INTRODUCTION}

Recently, differential equations with piecewise constant arguments (EPCA) has received much attention from a number of investigators $[1,2,3,4]$ in such various fields as population dynamics, physics, mechanical systems and control science. The theory of EPCA was initiated in 1983 and 1984 with the contributions of Wiener [5], Shah and Wiener [6] and Cooke and Wiener [7] and has been developed by many authors [8, 9, 10, 11]. In 1993, Wiener, pioneer of EPCA recollected in the book [12] the investigation of EPCA until that moment. Later, continuous efforts have been devoted to considering various properties of EPCA $[13,14,15,16,17,18]$. However, all of them are based on ordinary differential equations (ODEs). As far as authors' knowledge goes, only few results are presented in the consideration of partial differential equations with piecewise constant arguments (PEPCA). With regard to the PEPCA, it has been argued in [19] that they naturally arise in the process of approximating PDEs by piecewise constant arguments. Thus, it is important to investigate boundary value problems (BVP) and initial-value problems (IVP) for EPCA with partial derivatives, and explore the influence of certain discontinuous delays on the behavior of solutions to some typical problems of mathematical physics.

(c)2018 Asia Pacific Journal of Mathematics 
The equation

$$
u_{t}(x, t)=a^{2} u_{x x}(x, t)-b u(x, t)
$$

describes heat flow in a rod with both diffusion $a^{2} u_{x x}$ along the rod and heat loss (or gain) across the lateral sides of the rod. Measuring the lateral heat change at discrete moments of time leads to the equation with piecewise continuous delay

$$
u_{t}(x, t)=a^{2} u_{x x}(x, t)-b u(x,[t])
$$

which was investigated in [19].

In 1992, Wiener and Debnath [20] considered two PEPCAs with [t], the qualitative properties of zero solution of these equations were studied. Moreover, they considered the equation of neutral type [21], and studied the behavior of the solutions. In [22, 23], the authors discussed the numerical stability of $\theta$-methods and Galerkin methods for PEPCA with $[t]$, respectively. In 2015, Veloz and Pinto [24] studied the PEPCA of generalized type, the existence, computability and stability for solutions were argued. Later, Bereketoglu and Lafci [25] investigated the existence, uniqueness, oscillation, instability and stability of the solutions of PEPCA with $[t-1]$.

In this paper, we consider the following initial boundary value problem

$$
\left\{\begin{array}{l}
u_{t}(x, t)=a^{2} u_{x x}(x, t)+b u_{x x}(x,[t])+c u_{x x}\left(x, 2\left[\frac{t+1}{2}\right]\right), \quad t>0, \\
u(0, t)=u(1, t)=0, \\
u(x, 0)=v(x),
\end{array}\right.
$$

where $a, b, c \in \mathbb{R}$ and $a \neq 0, u: \Omega=[0,1] \times[0, \infty) \rightarrow \mathbb{R}, v:[0,1] \rightarrow \mathbb{R},[\cdot]$ signifies the greatest integer function. Our main object is to study the stability of analytic solution of (1). From the view point of structure in equation, our result is the generalization of the existing ones.

\section{THE STABILITY OF THE ANALYTIC SOLUTION}

Definition 1. [12] A solution of (1) is a function $u(x, t)$ satisfies the conditions:

(i) $u(x, t)$ is continuous in $\Omega$,

(ii) The partial derivative $u_{t}, u_{x}, u_{x x}$ exist and are continuous in $\Omega$ with the possible exception of the points $(x, n)(n=1,2, \cdots)$, where one-sided derivatives exist,

(iii) $u(x, t)$ satisfies $u_{t}(x, t)=a^{2} u_{x x}(x, t)+b u_{x x}(x,[t])+c u_{x x}(x, 2[(t+1) / 2]), t>0$ in $\Omega$ with the possible exception of the points $(x, n)$, and conditions $u(0, t)=u(1, t)=0$ and $u(x, 0)=v(x)$.

Definition 2. [12] If any solution $u(x, t)$ of (1) satisfies

$$
\lim _{t \rightarrow \infty} u(x, t)=0, x \in[0,1],
$$


then the zero solution of (1) is called asymptotically stable.

In order to derive the stability conditions of (1), we consider the following problem

$$
\left\{\begin{array}{l}
\mathbf{U}_{t}(x, t)=\mathbf{A} \mathbf{U}_{x x}(x, t)+\mathbf{B} \mathbf{U}_{x x}(x,[t])+\mathbf{C U}_{x x}\left(x, 2\left[\frac{t+1}{2}\right]\right), \quad t>0, \\
\mathbf{U}(0, t)=\mathbf{U}(1, t)=\mathbf{0}, \\
\mathbf{U}(x, 0)=\mathbf{U}_{0}(x)
\end{array}\right.
$$

where $\mathbf{U}(x, t)$ and $\mathbf{U}_{0}(x)$ are real $m \times m$ matrix, $\mathbf{A}, \mathbf{B}$ and $\mathbf{C}$ are real constant $m \times m$ matrix, by the method of separation of variables, it is not difficult to know the nonzero solution of (2) is

$$
\mathbf{U}(x, t)=\mathbf{T}(t) \mathbf{X}(x),
$$

so the equation in (2) gives

$$
\mathbf{T}^{\prime}(t) \mathbf{X}(x)=\mathbf{A} \mathbf{T}(t) \mathbf{X}^{\prime \prime}(x)+\mathbf{B} \mathbf{T}([t]) \mathbf{X}^{\prime \prime}(x)+\mathbf{C} \mathbf{T}\left(2\left[\frac{t+1}{2}\right]\right) \mathbf{X}^{\prime \prime}(x),
$$

i.e.,

$$
\left(\mathbf{A T}(t)+\mathbf{B T}([t])+\mathbf{C T}\left(2\left[\frac{t+1}{2}\right]\right)\right)^{-1} \mathbf{T}^{\prime}(t)=\mathbf{X}^{\prime \prime}(x)(\mathbf{X}(x))^{-1},
$$

we set

$$
\left(\mathbf{A T}(t)+\mathbf{B T}([t])+\mathbf{C T}\left(2\left[\frac{t+1}{2}\right]\right)\right)^{-1} \mathbf{T}^{\prime}(t)=\mathbf{X}^{\prime \prime}(x)(\mathbf{X}(x))^{-1}=-\mathbf{P}^{2},
$$

which gives the BVP

$$
\left\{\begin{array}{l}
\mathbf{X}^{\prime \prime}(x)+\mathbf{P}^{2} \mathbf{X}(x)=\mathbf{0} \\
\mathbf{X}(0)=\mathbf{X}(1)=\mathbf{0}
\end{array}\right.
$$

and equation on time $t$

$$
\mathbf{T}^{\prime}(t)+\mathbf{A} \mathbf{T}(t) \mathbf{P}^{2}+\mathbf{B T}([t]) \mathbf{P}^{2}+\mathbf{C T}\left(2\left[\frac{t+1}{2}\right]\right) \mathbf{P}^{2}=\mathbf{0} .
$$

The general solution of (3) is

$$
\mathbf{X}(x)=\mathbf{C}_{1} \cos (x \mathbf{P})+\mathbf{C}_{2} \sin (x \mathbf{P}),
$$

where

$$
\cos (x \mathbf{P})=\sum_{n=0}^{\infty} \frac{(-1)^{n} x^{2 n} \mathbf{P}^{2 n}}{(2 n) !}, \quad \sin (x \mathbf{P})=\sum_{n=0}^{\infty} \frac{(-1)^{n} x^{2 n+1} \mathbf{P}^{2 n+1}}{(2 n+1) !},
$$

and $\mathbf{C}_{1}, \mathbf{C}_{2}$ are arbitrary constant matrices.

From $\mathbf{X}(0)=\mathbf{0}$ we get that $\mathbf{C}_{1}=\mathbf{0}$, and $\mathbf{X}(1)=\mathbf{0}$ gives $\sin \mathbf{P}=0$, thus

$$
\mathbf{P}_{\mathbf{j}}=\operatorname{diag}(\pi(m(j-1)+1), \cdots, \pi m j), \quad j=1,2, \cdots, m,
$$

so we have the following result. 
Theorem 1. There exists an infinite sequence of matrix eigenfunctions for (3)

$$
\mathbf{X}_{j}(x)=\sqrt{2} \operatorname{diag}(\sin \pi(m(j-1)+1) x, \cdots, \sin \pi m j x), \quad j=1,2, \cdots, m .
$$

The solution of (4) will be derived in the following theorem.

Theorem 2. Let $\mathbf{E}(t)$ be the solution of the problem

$$
\mathbf{T}^{\prime}(t)=-\mathbf{A T}(t) \mathbf{P}^{2}, \quad \mathbf{T}(0)=\mathbf{I},
$$

and let

$$
\mathbf{M}(t)=\mathbf{E}(t)+(\mathbf{E}(t)-\mathbf{I}) \mathbf{A}^{-1} \mathbf{B}, \quad \mathbf{N}(t)=(\mathbf{E}(t)-\mathbf{I}) \mathbf{A}^{-1} \mathbf{C},
$$

if the matrix $\mathbf{A}$ is nonsingular, then the problem

$$
\left\{\begin{array}{l}
\mathbf{T}^{\prime}(t)+\mathbf{A T}(t) \mathbf{P}^{2}+\mathbf{B T}([t]) \mathbf{P}^{2}+\mathbf{C T}\left(2\left[\frac{t+1}{2}\right]\right) \mathbf{P}^{2}=\mathbf{0}, \quad t \geq 0 \\
\mathbf{T}(0)=\mathbf{C}_{0}
\end{array}\right.
$$

has a unique solution

$$
\begin{aligned}
\mathbf{T}(t)= & \mathbf{M}\left(t-2\left[\frac{t+1}{2}\right]+1\right)\left((I-\mathbf{N}(1))^{-1} \mathbf{M}(1)\right)^{-1} \\
& \left(\mathbf{M}(2)+\mathbf{N}(2)[I-\mathbf{N}(1)]^{-1} \mathbf{M}(1)\right)^{\left[\frac{t+1}{2}\right]} \mathbf{C}_{0} \\
& +\mathbf{N}\left(t-2\left[\frac{t+1}{2}\right]+1\right)\left(\mathbf{M}(2)+\mathbf{N}(2)(I-\mathbf{N}(1))^{-1} \mathbf{M}(1)\right)^{\left[\frac{t+1}{2}\right]} \mathbf{C}_{0} .
\end{aligned}
$$

Proof: On the interval $[2 n-1,2 n+1)$, it follows (4) we have

$$
\begin{aligned}
\mathbf{T}^{\prime}(t) & =-\mathbf{A T}(t) \mathbf{P}^{2}-\mathbf{B T}(2 n-1) \mathbf{P}^{2}-\mathbf{C T}(2 n) \mathbf{P}^{2} \\
& =-\mathbf{A T}(t) \mathbf{P}^{2}-\mathbf{B} \mathbf{C}_{2 n-1} \mathbf{P}^{2}-\mathbf{C C}_{2 n} \mathbf{P}^{2},
\end{aligned}
$$

with the general solution

$$
\mathbf{T}(t)=\mathbf{E}(t-2 n+1) \mathbf{K}-\mathbf{A}^{-1} \mathbf{B C}_{2 n-1}-\mathbf{A}^{-1} \mathbf{C C}_{2 n},
$$

at $t=2 n-1$

$$
\mathbf{C}_{2 n-1}=\mathbf{K}-\mathbf{A}^{-1} \mathbf{B C}_{2 n-1}-\mathbf{A}^{-1} \mathbf{C C}_{2 n},
$$

hence we obtain

$$
\mathbf{K}=\mathbf{C}_{2 n-1}+\mathbf{A}^{-1} \mathbf{B C}_{2 n-1}+\mathbf{A}^{-1} \mathbf{C C}_{2 n},
$$

thus we have

$$
\mathbf{T}(t)=\left\{\mathbf{E}(t-2 n+1)+(\mathbf{E}(t-2 n+1)-\mathbf{I}) \mathbf{A}^{-1} \mathbf{B}\right\} \mathbf{C}_{2 n-1}+(\mathbf{E}(t-2 n+1)-\mathbf{I}) \mathbf{A}^{-1} \mathbf{C C}_{2 n},
$$

which is equivalent to

$$
\mathbf{T}(t)=\mathbf{M}(t-2 n+1) \mathbf{C}_{2 n-1}+\mathbf{N}(t-2 n+1) \mathbf{C}_{2 n},
$$

at $t=2 n$

$$
\mathbf{C}_{2 n}=\mathbf{M}(1) \mathbf{C}_{2 n-1}+\mathbf{N}(1) \mathbf{C}_{2 n}
$$


so we derive

$$
\mathbf{C}_{2 n}=(I-\mathbf{N}(1))^{-1} \mathbf{M}(1) \mathbf{C}_{2 n-1},
$$

on the other hand, at $t=2 n+1$

$$
\mathbf{C}_{2 n+1}=\mathbf{M}(2) \mathbf{C}_{2 n-1}+\mathbf{N}(2) \mathbf{C}_{2 n}=\left(\mathbf{M}(2)+\mathbf{N}(2)[I-\mathbf{N}(1)]^{-1} \mathbf{M}(1)\right) \mathbf{C}_{2 n-1},
$$

then

$$
\mathbf{C}_{2 n+1}=\left(\mathbf{M}(2)+\mathbf{N}(2)(I-\mathbf{N}(1))^{-1} \mathbf{M}(1)\right)^{n} \mathbf{C}_{1},
$$

so

$$
\begin{gathered}
\mathbf{C}_{2 n}=\left(\mathbf{M}(2)+\mathbf{N}(2)(I-\mathbf{N}(1))^{-1} \mathbf{M}(1)\right)^{n} \mathbf{C}_{0} . \\
\mathbf{C}_{2 n-1}=\left((I-\mathbf{N}(1))^{-1} \mathbf{M}(1)\right)^{-1}\left(\mathbf{M}(2)+\mathbf{N}(2)(I-\mathbf{N}(1))^{-1} \mathbf{M}(1)\right)^{n} \mathbf{C}_{0} .
\end{gathered}
$$

From them it is immediate that

$$
\begin{aligned}
\mathbf{T}(t)= & \mathbf{M}(t-2 n+1) \mathbf{C}_{2 n-1}+\mathbf{N}(t-2 n+1) \mathbf{C}_{2 n} \\
= & \mathbf{M}(t-2 n+1)\left((I-\mathbf{N}(1))^{-1} \mathbf{M}(1)\right)^{-1} \\
& \left(\mathbf{M}(2)+\mathbf{N}(2)(I-\mathbf{N}(1))^{-1} \mathbf{M}(1)\right)^{n} \mathbf{C}_{0} \\
& +\mathbf{N}(t-2 n+1)\left(\mathbf{M}(2)+\mathbf{N}(2)(I-\mathbf{N}(1))^{-1} \mathbf{M}(1)\right)^{n} \mathbf{C}_{0} \\
= & \mathbf{M}\left(t-2\left[\frac{t+1}{2}\right]+1\right)\left((I-\mathbf{N}(1))^{-1} \mathbf{M}(1)\right)^{-1} \\
& \left(\mathbf{M}(2)+\mathbf{N}(2)(I-\mathbf{N}(1))^{-1} \mathbf{M}(1)\right)^{\left[\frac{t+1}{2}\right]} \mathbf{C}_{0} \\
& +\mathbf{N}\left(t-2\left[\frac{t+1}{2}\right]+1\right)\left(\mathbf{M}(2)+\mathbf{N}(2)(I-\mathbf{N}(1))^{-1} \mathbf{M}(1)\right)^{\left[\frac{t+1}{2}\right]} \mathbf{C}_{0},
\end{aligned}
$$

the proof is finished.

Thus the main result is as follows.

Corollary 1. If the following conditions are satisfied

$$
\begin{aligned}
& \left(a^{2}+b+c\right)\left(\left(a^{2}+b-c\right) e^{-a^{2} \pi^{2} j^{2}}-\left(b-a^{2}-c\right)\right)>0, \\
& \left(a^{2}+b+c\right)\left(\left(a^{2}+b+c\right) e^{-a^{2} \pi^{2} j^{2}}-\left(b-a^{2}+c\right)\right)>0,
\end{aligned}
$$

where

$$
c \neq \frac{a^{2}}{e^{-a^{2} \pi^{2} j^{2}}-1}, \quad a \neq 0
$$

then the zero solution of (1) is asymptotically stable.

Proof: If $a \neq 0$, let

$$
m=1, \mathbf{A}=a^{2}, \mathbf{P}=P_{j}=\pi j,
$$

in view of Theorems 1 and 2, we have

$$
\begin{aligned}
& E_{j}(t)=e^{-a^{2} \pi^{2} j^{2} t} \\
& M_{j}(t)=e^{-a^{2} \pi^{2} j^{2} t}+\frac{b}{a^{2}}\left(e^{-a^{2} \pi^{2} j^{2} t}-1\right), \\
& N_{j}(t)=\frac{c}{a^{2}}\left(e^{-a^{2} \pi^{2} j^{2} t}-1\right) .
\end{aligned}
$$


The zero solution of (1) is asymptotically stable if and only if

$$
\left|\begin{array}{l}
\frac{e^{-a^{2} \pi^{2} j^{2}}+\frac{b}{a^{2}}\left(e^{-a^{2} \pi^{2} j^{2}}-1\right)}{1-\frac{c}{a^{2}}\left(e^{-a^{2} \pi^{2} j^{2}}-1\right)} \mid<1, \\
e^{-a^{2} \pi^{2} j^{2}}+\frac{b+c}{a^{2}}\left(e^{-a^{2} \pi^{2} j^{2}}-1\right)
\end{array}\right|<1,
$$

which is equivalent to

$$
\begin{gathered}
\left(\frac{e^{-a^{2} \pi^{2} j^{2}}+\frac{b}{a^{2}}\left(e^{-a^{2} \pi^{2} j^{2}}-1\right)}{1-\frac{c}{a^{2}}\left(e^{-a^{2} \pi^{2} j^{2}}-1\right)}+1\right)\left(\frac{e^{-a^{2} \pi^{2} j^{2}}+\frac{b}{a^{2}}\left(e^{-a^{2} \pi^{2} j^{2}}-1\right)}{1-\frac{c}{a^{2}}\left(e^{-a^{2} \pi^{2} j^{2}}-1\right)}-1\right)<0, \\
\left(e^{-a^{2} \pi^{2} j^{2}}+\frac{b+c}{a^{2}}\left(e^{-a^{2} \pi^{2} j^{2}}-1\right)+1\right)\left(e^{-a^{2} \pi^{2} j^{2}}+\frac{b+c}{a^{2}}\left(e^{-a^{2} \pi^{2} j^{2}}-1\right)-1\right)<0 .
\end{gathered}
$$

After some deductions, we can get $(7)$.

\section{ACKNOWLEDGEMENTS}

This research is supported by the Natural Science Foundation of Guangdong Province (No. 2017A030313031).

\section{REFERENCES}

[1] F. Gurcan, F. Bozkurt, Global stability in a population model with piecewise constant arguments, J. Math. Anal. Appl. 360(2009), 334-342.

[2] L. Dai, M.C. Singh, On oscillatory motion of spring-mass systems subjected to piecewise constant forces, J. Sound Vib. 173(1994), 217-232.

[3] L. Dai, L. Fan, Analytical and numerical approaches to characteristics of linear and nonlinear vibratory systems under piecewise discontinuous disturbances, Commun. Nonlinear Sci. Numer. Simulat. 9(2004), 417-429.

[4] J. Wiener, V. Lakshmikantham, A damped oscillator with piecewise constant time delay, Nonlinear Stud. 1(2000), 78-84.

[5] J. Wiener, Differential equations with piecewise constant delays, in: V. Lakshmikantham (Ed.), Trends in the Theory and Practice of Nonlinear Differential Equations. Marcel Dekker, New York, 1983, 547580.

[6] S.M. Shah, J. Wiener, Advanced differential equations with piecewise constant argument deviations, Int. J. Math. Math. Sci. 6(1983), 671-703.

[7] K.L. Cooke, J. Wiener, Retarded differential equations with piecewise constant delays, J. Math. Anal. Appl. 99(1984), 265-297.

[8] Y.L. Lu, M.H. Song, M.Z. Liu, Convergence and stability of the split-step theta method for stochastic differential equations with piecewise continuous arguments, J. Comput. Appl. Math. 317(2017), 55-71.

[9] H. Bereketoglu, G. Seyhan, A. Ogun, Advanced impulsive differential equations with piecewise constant arguments, Math. Model. Anal. 15 (2010), 175-187.

[10] M.U. Akhmet, D. Arugăslanc, E. Y1lmaz, Stability in cellular neural networks with a piecewise constant argument, J. Comput. Appl. Math. 233(2010), 2365-2373. 
[11] M. Pinto, Asymptotic equivalence of nonlinear and quasi linear differential equations with piecewise constant arguments, Math. Comput. Model. 49(2009), 1750-1758.

[12] J. Wiener, Generalized Solutions of Functional Differential Equations, World Scientific, Singapore, 1993.

[13] Z.F. El Raheem, S.M. Salman, On a discretization process of fractional-order Logistic differential equation, J. Egyptian Math. Soc. 22(2014), 407-412.

[14] F. Cavalli, A. Naimzada, Amultiscale time model with piecewise constant argument for a boundedly rational monopolist, J. Differ. Equ. Appl. 22(2016), 1480-1489.

[15] W. Dimbour, Almost automorphic solutions for differential equations with piecewise constant argument in a Banach space, Nonlinear Anal. 74(2011), 2351-2357.

[16] L. Berezansky, E. Braverman, Stability conditions for scalar delay differential equations with a non-delay term, Appl. Math. Comput. 250(2015), 157-164.

[17] C. Li, C.J. Zhang, Block boundary value methods applied to functional differential equations with piecewise continuous arguments, Appl. Numer. Math. 115(2017), 214-224.

[18] M. Milosevic, The Euler-Maruyama approximation of solutions to stochastic differential equations with piecewise constant arguments, J. Comput. Appl. Math. 298(2016), 1-12.

[19] J. Wiener, Boundary value problems for partial differential equations with piecewise constant delay, Int. J. Math. Math. Sci. 14(1991), 363-379.

[20] J. Wiener, L. Debnath, A wave equation with discontinuous time delay, Int. J. Math. Math. Sci. 15(1992), 781-788.

[21] J. Wiener, L. Debnath, Boundary value problems for the diffusion equation with piecewise continuous time delay, Int. J. Math. Math. Sci. 20(1997), 187-195.

[22] H. Liang, M.Z. Liu, W.J. Lv, Stability of $\theta$-schemes in the numerical solution of a partial differential equation with piecewise continuous arguments, Appl. Math. Lett. 23(2010), 198-206.

[23] H. Liang, D.Y. Shi, W.J. Lv, Convergence and asymptotic stability of Galerkin methods for a partial differential equation with piecewise constant argument, Appl. Math. Comput. 217(2010), 854-860.

[24] T. Veloz, M. Pinto, Existence, computability and stability for solutions of the diffusion equation with general piecewise constant argument, J. Math. Anal. Appl. 426(2015), 330-339.

[25] H. Bereketoglu, M. Lafci, Behavior of the solutions of a partial differential equation with a piecewise constant argument, Filomat 31(2017), 5931-5943. 